\title{
COHEN-MACAULAY PROPERTIES UNDER THE AMALGAMATED CONSTRUCTION
}

\author{
Y. AZIMI, P. SAHANDI, AND N. SHIRMOHAMMADI
}

\begin{abstract}
Let $A$ and $B$ be commutative rings with unity, $f: A \rightarrow B$ a ring homomorphism and $J$ an ideal of $B$. Then the subring $A \bowtie^{f} J:=\{(a, f(a)+j) \mid a \in A$ and $j \in J\}$ of $A \times B$ is called the amalgamation of $A$ with $B$ along $J$ with respect to $f$. In this paper, we study the property of CohenMacaulay in the sense of ideals which was introduced by Asgharzadeh and Tousi, a general notion of the usual Cohen-Macaulay property (in the Noetherian case), on the ring $A \bowtie^{f} J$. Among other things, we obtain a generalization of the well-known result that when the Nagata's idealization is Cohen-Macaulay.
\end{abstract}

\section{INTRODUCTION}

The theory of Cohen-Macaulay rings is a major area of study in commutative algebra and algebraic geometry. From the appearance of the notion of Cohen-Macaulayness, this notion admits a rich theory in commutative Noetherian rings. There have been attempts to extend this notion to commutative non-Noetherian rings, since Glaz raised the question that whether there exists a generalization of the notion of Cohen-Macaulayness with certain desirable properties to nonNoetherian rings [13], [14]. In order to provide an answer to the question of Glaz [14, Page 220], recently several notions of Cohen-Macaulayness for non-Noetherian rings and modules were introduced in [15], [16], and [2]. Among those is the Cohen-Macaulay in the sense of $\mathscr{A}$, introduced by Asgharzadeh and Tousi [2], where $\mathscr{A}$ is a non-empty subclass of ideals of a commutative ring (the definition will be given later in Section 2).

In [7] and [8], D' Anna, Finocchiaro, and Fontana have introduced the following new ring construction. Let $A$ and $B$ be commutative rings with unity, let $J$ be an ideal of $B$ and let $f: A \rightarrow B$ be a ring homomorphism. The amalgamation of $A$ with $B$ along $J$ with respect to $f$ is the following subring

$$
A \bowtie^{f} J:=\{(a, f(a)+j) \mid a \in A \text { and } j \in J\}
$$

of $A \times B$. This construction generalizes the amalgamated duplication of a ring along an ideal (introduced and studied in [6], [10]). Moreover, several classical constructions such as the Nagata's idealization (cf. [19, page 2], [17, Chapter VI, Section 25]), the $A+X B[X]$ and the $A+X B[[X]]$ constructions can be studied as particular cases of this new construction (see [7, Examples 2.5 and 2.6]).

Below, we review briefly some known results about the behavior of Cohen-Macaulayness under the amalgamated construction and its particular cases.

Let $M$ be an $A$-module. In 1955, Nagata introduced a ring extension of $A$ called the trivial extension of $A$ by $M$ (or the idealization of $M$ in $A$ ), denoted here by $A \ltimes M$. Now, assume that $A$ is Noetherian local and that $M$ is finitely generated. It is well known that the trivial extension $A \ltimes M$ is Cohen-Macaulay if and only if $A$ is Cohen-Macaulay and $M$ is maximal Cohen-Macaulay, see [1, Corollary 4.14].

2010 Mathematics Subject Classification. 13A15, 13C14, $13 \mathrm{C} 15$.

Key words and phrases. Amalgamated algebra, Cohen-Macaulay ring, Koszul grade, Non-Noetherian ring. 
Let $A$ be a Noetherian local ring and $I$ be an ideal of $A$. Consider the amalgamated duplication $A \bowtie I:=\{(a, a+i) \mid a \in A$ and $i \in I\}$ as in [6], [10]. The properties of being Cohen-Macaulay, generalized Cohen-Macaulay, Gorenstein, quasi-Gorenstein, $\left(S_{n}\right),\left(R_{n}\right)$ and normality under the construction of amalgamated duplication were studied further in many research papers such as [6], [9], [3], and [21].

In [9], under the condition that $A$ is Cohen-Macaulay (Noetherian local) and $J$ is a finitely generated $A$-module, it is observed that $A \bowtie^{f} J$ is a Cohen-Macaulay ring if and only if it is a Cohen-Macaulay $A$-module if and only if $J$ is a maximal Cohen-Macaulay module. Then, in [22], assuming $(A, \mathfrak{m})$ is Noetherian local, $J$ is contained in the Jacobson radical of $B$ such that $\operatorname{depth}_{A} J<\infty$ and that $f^{-1}(\mathfrak{q}) \neq \mathfrak{m}$, for each $\mathfrak{q} \in \operatorname{Spec}(B) \backslash V(J)$, it is shown that $A \bowtie^{f} J$ is CohenMacaulay if and only if $A$ is Cohen-Macaulay and $J$ is a big Cohen-Macaulay $A$-module (i.e. $\operatorname{depth}_{A} J=\operatorname{dim} A$ ).

The next natural step is to seek when the amalgamated algebra $A \bowtie^{f} J$ is Cohen-Macaulay without Noetherian assumption.

In this paper, we investigate the property of Cohen-Macaulay in the sense of ideals (resp. maximal ideals, finitely generated ideals) on the amalgamation. More precisely, in Section 2, we recall some essential definitions and results on which we base our approach. In Section 3, we fix our notation and give some elementary results on the behavior of the Koszul grade with respect to amalgamation. In Section 4, we classify some necessary and sufficient conditions for the amalgamated algebra $A \bowtie^{f} J$ to be Cohen-Macaulay in the sense of ideals (resp. maximal ideals, finitely generated ideals) (Theorems 4.1,4.6 and 4.11). Among the applications of our results are the classification of when the trivial extension $A \ltimes M$ and the amalgamated duplication $A \bowtie I$ are Cohen-Macaulay in the sense of ideals (Corollaries 4.8 and 4.16).

\section{PRELIMINARIES}

To facilitate the reading of the paper, we recall in this section some preliminary definitions and properties to be used later.

Let $\mathfrak{b}$ be a finitely generated ideal of a commutative $\operatorname{ring} A$ and $M$ be an $A$-module. Assume that $\mathfrak{b}$ is generated by the sequence $\mathbf{x}=x_{1}, \ldots, x_{\ell}$. We denote the Koszul complex related to $\mathbf{x}$ by $\mathbb{K}_{\bullet}(\mathbf{x})$. The Koszul grade of $\mathfrak{b}$ on $M$ is defined by

$$
\mathrm{K} \cdot \operatorname{grade}_{A}(\mathfrak{b}, M):=\inf \left\{i \in \mathbb{N} \cup\{0\} \mid H^{i}\left(\operatorname{Hom}_{A}\left(\mathbb{K}_{\bullet}(\mathbf{x}), M\right)\right) \neq 0\right\} .
$$

It follows from [5, Corollary 1.6.22] and [5, Proposition 1.6.10(d)] that this does not depend on the choice of generating sets of $\mathfrak{b}$.

Let $\mathfrak{a}$ be an arbitrary ideal of $A$. One can then define the Koszul grade of $\mathfrak{a}$ on $M$ by setting

$$
\mathrm{K} \cdot \operatorname{grade}_{A}(\mathfrak{a}, M):=\sup \left\{\mathrm{K} \cdot \operatorname{grade}_{A}(\mathfrak{b}, M) \mid \mathfrak{b} \text { is a finitely generated subideal of } \mathfrak{a}\right\} \text {. }
$$

In view of [5, Proposition 9.1.2(f)], this definition coincides with the original one for finitely generated ideals. In particular, when $(A, \mathfrak{m})$ is local Noetherian, $\operatorname{depth}_{A} M$ was defined by $\operatorname{K}_{\cdot} \operatorname{grade}_{A}(\mathfrak{m}, M)$ in [5, Section 9.1].

The $\check{C}$ ech grade of $\mathfrak{b}$ on $M$ is defined by

$$
\check{\mathrm{C}} . \operatorname{grade}_{A}(\mathfrak{b}, M):=\inf \left\{i \in \mathbb{N} \cup\{0\} \mid H_{\mathbf{x}}^{i}(M) \neq 0\right\} .
$$

Here $H_{\mathbf{x}}^{i}(M)$ denotes the $i$-th cohomology of the $\check{C} e c h$ complex of $M$ related to $\mathbf{x}$. It follows from [16, Proposition 2.1(e)] that $H_{\mathbf{x}}^{i}(M)$ is independent of the choice of sequence of generators for $\mathfrak{b}$. One can then define

$$
\check{\mathrm{C}} \cdot \operatorname{grade}_{A}(\mathfrak{a}, M):=\sup \left\{\check{\mathrm{C}} \cdot \operatorname{grade}_{A}(\mathfrak{b}, M) \mid \mathfrak{b} \text { is a finitely generated subideal of } \mathfrak{a}\right\} \text {. }
$$

By virtue of [16, Proposition 2.7], one has $\check{C} \cdot \operatorname{grade}_{A}(\mathfrak{a}, M)=\mathrm{K} \cdot \operatorname{grade}_{A}(\mathfrak{a}, M)$. 
Let $\mathfrak{p}$ a prime ideal of $R$. By ht $M \mathfrak{p}$, we mean the Krull dimension of the $R_{\mathfrak{p}}$-module $M_{\mathfrak{p}}$. Also,

$$
\mathrm{ht}_{M} \mathfrak{a}:=\inf \left\{\mathrm{ht}_{M} \mathfrak{p} \mid \mathfrak{p} \in \operatorname{Supp}_{A}(M) \cap V(\mathfrak{a})\right\} .
$$

Let $\mathscr{A}$ be a non-empty subclass of the class of all ideals of the ring $A$ and $M$ be an $A$-module. We say that $M$ is Cohen-Macaulay in the sense of $\mathscr{A}$ if ht $_{M}(\mathfrak{a})=\mathrm{K} \cdot \operatorname{grade}_{A}(\mathfrak{a}, M)$ for all ideals $\mathfrak{a}$ in $\mathscr{A}$, see [2, Definition 3.1]. The classes we are interested in are the class of all maximal ideals, the class of all ideals and the class of all finitely generated ideals. Assume that $A$ is Noetherian. It is well-known that $A$ is Cohen-Macaulay (in the sense of the original definition in the Noetherian setting) if and only if it is Cohen-Macaulay in the sense of ideals (resp. maximal ideals, finitely generated ideals) see [5, Corollary 2.1.4].

\section{The Koszul gRAde on Amalgamation}

Let us fix some notation which we shall use frequently throughout the paper: $A, B$ are two commutative rings with unity, $f: A \rightarrow B$ is a ring homomorphism, and $J$ denotes an ideal of $B$. So that $J$ is an $A$-module via the homomorphism $f$. In the sequel, we consider the contraction and extension with respect to the natural embedding $\imath_{A}: A \rightarrow A \bowtie^{f} J$ defined by $\imath_{A}(x)=(x, f(x))$, for every $x \in A$. In particular, for every ideal $\mathfrak{a}$ of $A, \mathfrak{a}^{e}$ means $\mathfrak{a}\left(A \bowtie^{f} J\right)$.

This section is devoted to prove some lemmas on the behavior of the Koszul grade on amalgamation. These lemmas provide the key for some crucial arguments later in this paper. In the proof of the next lemma, we use $H_{i}(\mathbf{x}, M)$ to denote the $i$ th Koszul homology of an $A$-module $M$ with respect to a finite sequence $\mathbf{x} \subset A$.

Lemma 3.1. Let the notation and hypotheses be as in the beginning of this section. Then

(i) for any finitely generated ideal $\mathfrak{b}$ of $A$, one has the equality

$$
\mathrm{K} \cdot \operatorname{grade}_{A \bowtie J}\left(\mathfrak{b}^{e}, A \bowtie^{f} J\right)=\min \left\{\mathrm{K} \cdot \operatorname{grade}_{A}(\mathfrak{b}, A), \mathrm{K} \cdot \operatorname{grade}_{A}(\mathfrak{b}, J)\right\} .
$$

(ii) for any ideal $\mathfrak{a}$ of $A$, one has the inequality

$$
\mathrm{K} \cdot \operatorname{grade}_{A \bowtie J}\left(\mathfrak{a}^{e}, A \bowtie^{f} J\right) \leq \min \left\{\mathrm{K} \cdot \operatorname{grade}_{A}(\mathfrak{a}, A), \mathrm{K} \cdot \operatorname{grade}_{A}(\mathfrak{a}, J)\right\} .
$$

Proof. Assume that $\mathfrak{b}$ is a finitely generated ideal of $A$ and that $\mathfrak{b}$ is generated by a finite sequence $\mathbf{x}$ of length $\ell$. Then, using [2, Proposition 2.2(iv)] together with [16, Proposition 2.7], we have

$$
\begin{aligned}
& \mathrm{K} \cdot \operatorname{grade}_{A \bowtie^{f} J}\left(\mathfrak{b}^{e}, A \bowtie^{f} J\right) \\
= & \mathrm{K} \cdot \operatorname{grade}_{A}\left(\mathfrak{b}, A \bowtie^{f} J\right) \\
= & \sup \left\{k \geq 0 \mid H_{\ell-i}\left(\mathbf{x}, A \bowtie^{f} J\right)=0 \text { for all } i<k\right\} \\
= & \sup \left\{k \geq 0 \mid H_{\ell-i}(\mathbf{x}, A) \oplus H_{\ell-i}(\mathbf{x}, J)=0 \text { for all } i<k\right\} \\
= & \min \left\{\mathrm{K}_{\cdot} \operatorname{grade}_{A}(\mathfrak{b}, A), \mathrm{K} \cdot \operatorname{grade}_{A}(\mathfrak{b}, J)\right\} .
\end{aligned}
$$

For the third equality, one notices that the amalgamation $A \bowtie^{f} J$, as an $A$-module, is isomorphic to the direct sum of $A \oplus J$ using [7, Lemma 2.3(4)]. This proves (1). To obtain (2), assume that $\mathfrak{a}$ is an ideal of $A$. Let $\Sigma$ be the class of all finitely generated subideals of $\mathfrak{a}$. It follows from the definition that

$$
\begin{aligned}
& \mathrm{K} \cdot \operatorname{grade}_{A}\left(\mathfrak{a}, A \bowtie^{f} J\right) \\
= & \sup \left\{\mathrm{K} \cdot \operatorname{grade}_{A}\left(\mathfrak{b}, A \bowtie^{f} J\right) \mid \mathfrak{b} \in \Sigma\right\} \\
= & \sup \left\{\min \left\{\mathrm{K} \cdot \operatorname{grade}_{A}(\mathfrak{b}, A), \mathrm{K} \cdot \operatorname{grade}_{A}(\mathfrak{b}, J)\right\} \mid \mathfrak{b} \in \Sigma\right\} \\
\leq & \min \left\{\sup \left\{\mathrm{K} \cdot \operatorname{grade}_{A}(\mathfrak{b}, A) \mid \mathfrak{b} \in \Sigma\right\}, \sup \left\{\mathrm{K} \cdot \operatorname{grade}_{A}(\mathfrak{b}, J) \mid \mathfrak{b} \in \Sigma\right\}\right\} \\
= & \min \left\{\mathrm{K} \cdot \operatorname{grade}_{A}(\mathfrak{a}, A), \mathrm{K} \cdot \operatorname{grade}_{A}(\mathfrak{a}, J)\right\} .
\end{aligned}
$$


Again, using this in conjunction with [2, Proposition 2.2(iv)], one deduces that

$$
\begin{aligned}
\mathrm{K} \cdot \operatorname{grade}_{A \bowtie f}\left(\mathfrak{a}^{e}, A \bowtie^{f} J\right) & =\mathrm{K} \cdot \operatorname{grade}_{A}\left(\mathfrak{a}, A \bowtie^{f} J\right) \\
& \leq \min \left\{\mathrm{K}_{\operatorname{grade}}(\mathfrak{a}, A), \mathrm{K} \cdot \operatorname{grade}_{A}(\mathfrak{a}, J)\right\} .
\end{aligned}
$$

Lemma 3.2. Assume that $A$ is Cohen-Macaulay in the sense of (finitely generated) ideals and $\mathrm{K}$. $\operatorname{grade}_{A}(\mathfrak{a}, J) \geq \mathrm{ht} \mathfrak{a}$ for every (finitely generated) ideal $\mathfrak{a}$ of $A$. Then

$$
\mathrm{K} \cdot \operatorname{grade}_{A \bowtie f}\left(\mathfrak{a}^{e}, A \bowtie^{f} J\right)=\mathrm{K} \cdot \operatorname{grade}_{A}(\mathfrak{a}, A) \leq \mathrm{K} \cdot \operatorname{grade}_{A}(\mathfrak{a}, J)
$$

for any (finitely generated) ideal $\mathfrak{a}$ of $A$.

Proof. Assume that $\mathfrak{a}$ is a (finitely generated) ideal of $A$ and let $\Sigma$ be the class of all finitely generated subideals of $\mathfrak{a}$. Then, as in the proof of Lemma 3.1, again, using [2, Proposition 2.2(iv)], we have

$$
\begin{aligned}
& \mathrm{K} \cdot \operatorname{grade}_{A \bowtie J}\left(\mathfrak{a}^{e}, A \bowtie^{f} J\right) \\
= & \mathrm{K} \cdot \operatorname{grade}_{A}\left(\mathfrak{a}, A \bowtie^{f} J\right) \\
= & \sup \left\{\mathrm{K} \cdot \operatorname{grade}_{A}\left(\mathfrak{b}, A \bowtie^{f} J\right) \mid \mathfrak{b} \in \Sigma\right\} \\
= & \sup \left\{\min \left\{\mathrm{K}_{\operatorname{grade}}(\mathfrak{b}, A), \mathrm{K} \cdot \operatorname{grade}_{A}(\mathfrak{b}, J)\right\} \mid \mathfrak{b} \in \Sigma\right\} \\
= & \sup \left\{\mathrm{K} \cdot \operatorname{grade}_{A}(\mathfrak{b}, A) \mid \mathfrak{b} \in \Sigma\right\} \\
= & \mathrm{K} \cdot \operatorname{grade}_{A}(\mathfrak{a}, A) .
\end{aligned}
$$

The forth equality follows from [2, Lemma 3.2] and our assumption. This completes the proof.

The following lemma is a slight modification of [2, Lemma 3.2].

Lemma 3.3. Let $\mathfrak{a}$ be an ideal of $A$ and $M$ be an A-module.

(i) Let $A$ be quasi-local with the maximal ideal $\mathfrak{m}$. If $\mathrm{K} \cdot \operatorname{grade}_{A}(\mathfrak{m}, M)<\infty$, then $\mathrm{K} \cdot \operatorname{grade}_{A}(\mathfrak{m}, M) \leq$ $\operatorname{dim} A$.

(ii) If, for every minimal prime ideal $\mathfrak{p}$ over $\mathfrak{a}, \mathrm{K} \cdot \operatorname{grade}_{A}\left(\mathfrak{p} R_{\mathfrak{p}}, M_{\mathfrak{p}}\right)<\infty($ e.g. when $M$ is finitely generated), then $\mathrm{K} \cdot \operatorname{grade}_{A}(\mathfrak{a}, M) \leq \mathrm{ht} \mathfrak{a}$.

Proof. (1) Using [16, Proposition 2.7], it is enough for us to show that $\check{C} \cdot \operatorname{grade}_{A}(\mathfrak{m}, M) \leq \operatorname{dim} A$. In order to prove this, assume that $\operatorname{dim} A<\infty$ and let $\mathbf{x}$ be a finite sequence of elements in $\mathfrak{m}$. It follows from [16, Proposition 2.4] that $\check{C} \cdot \operatorname{grade}_{A}(\mathbf{x}, M) \leq \operatorname{dim} A$. Therefore $\check{C} \cdot \operatorname{grade}_{A}(\mathfrak{m}, M) \leq \operatorname{dim} A$. (2) Notice, by [2, Proposition 2.2(iii)], that K. $\operatorname{grade}_{A}(\mathfrak{a}, M)<\infty$. Then, by [2, Proposition 2.2(ii) and (iii)], one may assume that $A$ is quasi-local with the maximal ideal $\mathfrak{m}$. Now (1) completes the proof.

\section{MAIN RESUltS}

Assume that $A$ is Noetherian local, and that $J$ is contained in the Jacobson radical of $B$ and it is a finitely generated $A$-module. Recall that a finitely generated module $M$ over $A$ is called a maximal Cohen-Macaulay A-module if $\operatorname{depth}_{A} M=\operatorname{dim} A$. Note that, in this circumstance, $\operatorname{depth}_{A} M$ equals the common length of the maximal $M$-regular sequences in the maximal ideal of $A$. In [22, Corollary 2.5], it is shown that $A \bowtie^{f} J$ is Cohen-Macaulay if and only if $A$ is Cohen-Macaulay and $J$ is a maximal Cohen-Macaulay $A$-module. Our first main result improves this corollary by removing the Noetherian assumption.

The reader should be aware that when we say $A \bowtie^{f} J$ is Cohen-Macaulay in the sense of a non-empty class of ideals, we mean $A \bowtie^{f} J$ is Cohen-Macaulay as a ring. 
Theorem 4.1. Assume that $(A, \mathfrak{m})$ is quasi-local such that $\mathfrak{m}$ is finitely generated. Assume that $J$ is contained in the Jacobson radical of $B$ and it is finitely generated as an A-module. Then $A \bowtie^{f} J$ is Cohen-Macaulay (ring) in the sense of maximal ideals if and only if $A$ is Cohen-Macaulay in the sense of maximal ideals and $\mathrm{K} \cdot \operatorname{grade}_{A}(\mathfrak{m}, J)=\operatorname{dim} A$.

Proof. Assume that $\mathfrak{m}$ is generated by the sequence $\mathbf{a}=a_{1}, \ldots, a_{n}$ and that $J$ is generated by the sequence $\mathbf{b}=b_{1}, \ldots, b_{m}$. Hence $\mathfrak{m}^{\prime} f=\mathfrak{m} \bowtie^{f} J$, the unique maximal ideal of $A \bowtie^{f} J[9$, Corollary 2.7(3)], is generated by the sequence $\mathbf{c}=\left(a_{1}, f\left(a_{1}\right)\right), \ldots,\left(a_{n}, f\left(a_{n}\right)\right),\left(0, b_{1}\right), \ldots,\left(0, b_{m}\right)$. Notice, by [9. Corollary 3.2 and Remark 3.3], that one has $\sqrt{l_{A}(\mathbf{a})\left(A \bowtie^{f} J\right)}=\sqrt{\mathfrak{m}\left(A \bowtie^{f} J\right)}=\mathfrak{m}^{\prime} f=$ c $\left(A \bowtie^{f} J\right)$. Therefore

$$
\begin{aligned}
& \mathrm{K} \cdot \operatorname{grade}_{A \bowtie^{f} J}\left(\mathfrak{m}^{\prime}, A \bowtie^{f} J\right)=\check{\mathrm{C}} \cdot \operatorname{grade}_{A \bowtie^{f} J}\left(\mathfrak{m}^{\prime}, A \bowtie^{f} J\right) \\
& =\inf \left\{i \mid H_{\mathbf{c}}^{i}\left(A \bowtie^{f} J\right) \neq 0\right\} \\
& =\inf \left\{i \mid H_{l_{A}(\mathbf{a})}^{i}\left(A \bowtie^{f} J\right) \neq 0\right\} \\
& =\inf \left\{i \mid H_{\mathbf{a}}^{i}\left(A \bowtie^{f} J\right) \neq 0\right\} \\
& =\inf \left\{i \mid H_{\mathbf{a}}^{i}(A) \oplus H_{\mathbf{a}}^{i}(J) \neq 0\right\} \\
& =\min \left\{\check{\mathrm{C}} \cdot \operatorname{grade}_{A}(\mathfrak{m}, A), \check{\mathrm{C}} \cdot \operatorname{grade}_{A}(\mathfrak{m}, J)\right\} \\
& =\min \left\{\mathrm{K}_{\operatorname{grade}}(\mathfrak{m}, A), \mathrm{K} \cdot \operatorname{grade}_{A}(\mathfrak{m}, J)\right\} .
\end{aligned}
$$

The first equality obtains by [16, Proposition 2.7], the third equality follows from [16, Proposition 2.1(e)] in conjunction with $\sqrt{l_{A}(\mathbf{a})\left(A \bowtie^{f} J\right)}=\mathbf{c}\left(A \bowtie^{f} J\right)$, the forth equality deduces from [16, Proposition 2.1(f)], and the fifth equality holds since as an $A$-module $A \bowtie^{f} J \cong A \oplus J$ [7, Lemma 2.3(4)].

Consequently, the conclusion yields by the equality

$$
\mathrm{K} \cdot \operatorname{grade}_{A \bowtie f}\left(\mathfrak{m}^{\prime}, A \bowtie^{f} J\right)=\min \left\{\mathrm{K} \cdot \operatorname{grade}_{A}(\mathfrak{m}, A), \mathrm{K} \cdot \operatorname{grade}_{A}(\mathfrak{m}, J)\right\}
$$

together with $\operatorname{dim} A \bowtie^{f} J=\operatorname{dim} A$. This last equality holds true, since $A \bowtie^{f} J$ is integral over $A$ (see [8, Proposition 4.2]).

Corollary 4.2. (See [22, Corollary 2.5]) Assume that $A$ is Noetherian local, and that $J$ is contained in the Jacobson radical of $B$ and it is finitely generated as an A-module. Then $A \bowtie^{f} J$ is CohenMacaulay (ring) if and only if $A$ is Cohen-Macaulay and $J$ is a maximal Cohen-Macaulay Amodule.

The key to the next theorem is given by the following elementary lemmas. Their proofs are straightforward; so that we omit them. Recall from [9, Corollary 2.5] that the prime ideals of $A \bowtie^{f} J$ are of the type $\overline{\mathfrak{q}}^{f}$ or $\mathfrak{p}^{\prime}$, for $\mathfrak{q}$ varying in $\operatorname{Spec}(B) \backslash V(J)$ and $\mathfrak{p}$ in $\operatorname{Spec}(A)$, where

$$
\begin{aligned}
& \mathfrak{p}^{\prime}:=\mathfrak{p} \bowtie^{f} J:=\{(p, f(p)+j) \mid p \in \mathfrak{p}, j \in J\}, \\
& \overline{\mathfrak{q}}^{f}:=\{(a, f(a)+j) \mid a \in A, j \in J, f(a)+j \in \mathfrak{q}\} .
\end{aligned}
$$

Lemma 4.3. Assume that $\mathfrak{a}$ is an ideal of $A, \mathfrak{p}$ is a prime ideal of $A$ and that $\mathfrak{q}$ is a prime ideal of B. Then

(i) $\mathfrak{a}^{e} \subseteq \mathfrak{p}^{\prime}$ if and only if $\mathfrak{a} \subseteq \mathfrak{p}$.

(ii) $\mathfrak{a}^{e} \subseteq \overline{\mathfrak{q}}^{f}$ if and only if $f(\mathfrak{a}) \subseteq \mathfrak{q}$.

In the sequel, we use $\operatorname{Nil}(B)$ to denote the nil radical of the $\operatorname{ring} B$.

Lemma 4.4. Assume that $\mathfrak{a}$ is an ideal of $A, J \subseteq \operatorname{Nil}(B)$ and that $\mathfrak{p}$ is a prime ideal of $A$. Then 
(i) $\mathfrak{p} \in \operatorname{Min}(\mathfrak{a})$ if and only if $\mathfrak{p}^{\prime} f \in \operatorname{Min}\left(\mathfrak{a}^{e}\right)$.

(ii) ht $\mathfrak{a}=\mathrm{ht} \mathfrak{a}^{e}$.

(iii) $\operatorname{Min}\left(\mathfrak{p}^{e}\right)=\left\{\mathfrak{p}^{\prime f}\right\}$. In particular ht $\mathfrak{p}^{e}=\mathrm{ht} \mathfrak{p}^{\prime f}$.

Proposition 4.5. Let $\mathscr{A}$ be a non-empty class of ideals of $A$. Assume that $\mathrm{ht} \mathfrak{a}^{e} \geq \mathrm{ht} \mathfrak{a}$ for each $\mathfrak{a} \in \mathscr{A}$. If $A \bowtie^{f} J$ is Cohen-Macaulay (ring) in the sense of $\mathscr{A}^{e}:=\left\{\mathfrak{a}^{e} \mid \mathfrak{a} \in \mathscr{A}\right\}$, then $A$ is CohenMacaulay in the sense of $\mathscr{A}$ and $\mathrm{K}$. $\operatorname{grade}_{A}(\mathfrak{a}, J) \geq \mathrm{ht} \mathfrak{a}$ for each $\mathfrak{a} \in \mathscr{A}$.

Proof. Assume that $\mathfrak{a} \in \mathscr{A}$. Then, by Lemma3.1(2), we have

$$
\begin{aligned}
\mathrm{K} \cdot \operatorname{grade}_{A}(\mathfrak{a}, A) & \geq \mathrm{K} \cdot \operatorname{grade}_{A \bowtie f}\left(\mathfrak{a}^{e}, A \bowtie^{f} J\right) \\
& =\mathrm{ht} \mathfrak{a}^{e} \\
& \geq \mathrm{ht} \mathfrak{a} \\
& \geq \mathrm{K} \cdot \operatorname{grade}_{A}(\mathfrak{a}, A) .
\end{aligned}
$$

Thus K. $\operatorname{grade}_{A}(\mathfrak{a}, A)=$ ht $\mathfrak{a}$. This means that $A$ is Cohen-Macaulay in the sense of $\mathscr{A}$. Similarly, one obtains K. $\operatorname{grade}_{A}(\mathfrak{a}, J) \geq$ ht $\mathfrak{a}$.

It is not clear for us whether, in general, the inequality ht $\mathfrak{a}^{e} \geq$ ht $\mathfrak{a}$ holds for each $\mathfrak{a} \in \mathscr{A}$. However, under the assumption $J \subseteq \operatorname{Nil}(B)$, for each ideal $\mathfrak{a}$, one has the equality ht $\mathfrak{a}^{e}=$ ht $\mathfrak{a}$ by Lemma 4.4

The second main result of the paper is the following theorem.

Theorem 4.6. Assume that $J \subseteq \operatorname{Nil}(B)$. Then $A \bowtie^{f} J$ is Cohen-Macaulay (ring) in the sense of ideals if and only if $A$ is Cohen-Macaulay in the sense of ideals and $\mathrm{K} \cdot \operatorname{grade}_{A}(\mathfrak{a}, J) \geq \mathrm{ht} \mathfrak{a}$ for every ideal $\mathfrak{a}$ of $A$.

Proof. One implication follows from Proposition 4.5 and Lemma 4.4(2). Then, to prove the converse, assume that $A$ is Cohen-Macaulay in the sense of ideals and $\mathrm{K}$. $\operatorname{grade}_{A}(\mathfrak{a}, J) \geq \mathrm{ht} \mathfrak{a}$ for every ideal $\mathfrak{a}$ of $A$. Let $\mathfrak{a}$ be an ideal of $A$. First observe, by Lemmas 3.2 and 4.4(2), that

$$
\begin{aligned}
\mathrm{K} \cdot \operatorname{grade}_{A \bowtie J}\left(\mathfrak{a}^{e}, A \bowtie^{f} J\right) & =\mathrm{K} \cdot \operatorname{grade}_{A}(\mathfrak{a}, A) \\
& =\mathrm{ht} \mathfrak{a} \\
& =\mathrm{ht} \mathfrak{a}^{e} .
\end{aligned}
$$

Now, let $I$ be an arbitrary proper ideal of $A \bowtie^{f} J$. Then, by [20, Theorem 16 of Chapter 5], there exists a prime ideal $\mathscr{P}$ of $A \bowtie^{f} J$ containing $I$ such that $\mathrm{K}$. $\operatorname{grade}_{A \bowtie^{f} J}\left(I, A \bowtie^{f} J\right)=\mathrm{K}$. $\operatorname{grade}_{A \bowtie^{f} J}\left(\mathscr{P}, A \bowtie^{f}\right.$ $J)$. Notice that $\mathscr{P}=\mathfrak{p}^{\prime} f$ for some prime ideal $\mathfrak{p}$ of $A$ by [9, Corollaries 2.5 and 2.7]. Hence, by Lemma 4.4(3), one has

$$
\begin{aligned}
\mathrm{ht} I & \geq \mathrm{K} \cdot \operatorname{grade}_{A \bowtie^{f} J}\left(I, A \bowtie^{f} J\right) \\
& =\mathrm{K} \cdot \operatorname{grade}_{A \bowtie^{f} J}\left(\mathfrak{p}^{\prime}, A \bowtie^{f} J\right) \\
& \geq \mathrm{K} \cdot \operatorname{grade}_{A \bowtie^{f} J}\left(\mathfrak{p}^{e}, A \bowtie^{f} J\right) \\
& =\mathrm{ht} \mathfrak{p}^{e} \\
& =\mathrm{ht} \mathfrak{p}^{\prime} \\
& \geq \mathrm{ht} I .
\end{aligned}
$$

Therefore $A \bowtie^{f} J$ is Cohen-Macaulay in the sense of ideals.

The next example shows that, if, in the above theorem, the hypothesis $J \subseteq \operatorname{Nil}(B)$ is dropped, then the corresponding statement is no longer always true. 
Example 4.7. Let $k$ be a field and $X, Y$ are algebraically independent indeterminates over $k$. Set $A:=k[[X]], B:=k[[X, Y]]$ and let $J:=(X, Y)$. Let $f: A \rightarrow B$ be the inclusion. Note that $A$ is CohenMacaulay and $\mathrm{K} \cdot \operatorname{grade}_{A}(\mathfrak{a}, J)=\mathrm{ht} \mathfrak{a}$ for every ideal $\mathfrak{a}$ of $A$. Indeed, if $\mathfrak{a}$ is a non-zero proper ideal of $A$, and $a$ is a non-zero element of $\mathfrak{a}$, then one has

$$
1 \leq \mathrm{K} \cdot \operatorname{grade}_{A}(a A, J) \leq \mathrm{K} \cdot \operatorname{grade}_{A}(\mathfrak{a}, J) \leq \mathrm{ht}_{J} \mathfrak{a} \leq \mathrm{ht} \mathfrak{a} \leq 1 .
$$

The first and second inequalities follow from [5, Proposition 9.1.2(a),(f)], respectively. While the third inequality follows from Lemma 3.3 (ii), the others are obvious. However, $A \bowtie^{f} J$ which is isomorphic to $k[[X, Y, Z]] /(Y, Z) \cap(X-Y)$ is not Cohen-Macaulay.

Let $M$ be a $A$-module. Then $A \ltimes M$ denotes the trivial extension of $A$ by $M$. It should be noted that $0 \ltimes M$ is an ideal in $A \ltimes M$ and $(0 \ltimes M)^{2}=0$. As in [7, Example 2.8], if $B:=A \ltimes M$, $J:=0 \ltimes M$, and $f: A \rightarrow B$ be the natural embedding, then $A \bowtie^{f} J \cong A \ltimes M$. Hence the next result follows from Theorem 4.6. With it, we not only offer an application of Theorem 4.6, but we also provide a generalization of the well-known characterization of when the trivial extension is Cohen-Macaulay in the Noetherian (local) case, see [1, Corollary 4.14].

Corollary 4.8. Let $M$ be an A-module. Then $A \ltimes M$ is Cohen-Macaulay (ring) in the sense of ideals if and only if $A$ is Cohen-Macaulay in the sense of ideals and $\mathrm{K} \cdot \operatorname{grade}_{A}(\mathfrak{a}, M) \geq \mathrm{ht} \mathfrak{a}$ for every ideal $\mathfrak{a}$ of $A$.

Assume that $A$ is Noetherian. In [22, Corollary 2.7], the authors showed that $A$ is CohenMacaulay if $A \bowtie^{f} J$ is Cohen-Macaulay provided that $f^{-1}(\mathfrak{q}) \neq \mathfrak{m}$ for each $\mathfrak{q} \in \operatorname{Spec}(B) \backslash V(J)$ and each $\mathfrak{m} \in \operatorname{Max}(A)$. In the following corollary we improve the conclusion of the mentioned result in the circumstance that $J \subseteq \operatorname{Nil}(B)$.

Assume that $A$ is Noetherian and $M$ is a finitely generated $A$-module. It can be seen that ht $\mathfrak{a} \leq \operatorname{grade}_{A}(\mathfrak{a}, M)\left(=\mathrm{K} \cdot \operatorname{grade}_{A}(\mathfrak{a}, M)\right)$ for every ideal $\mathfrak{a}$ of $A$ if and only if $M_{\mathfrak{p}}$ is maximal CohenMacaulay for every prime ideal $\mathfrak{p} \in \operatorname{Supp}_{A}(M)$. Indeed, assume that $M_{\mathfrak{p}}$ is maximal CohenMacaulay for every prime ideal $\mathfrak{p} \in \operatorname{Supp}_{A}(M)$, and $\mathfrak{a}$ is an ideal of $R$. There is nothing to prove if $\mathfrak{a} M=M$, since in this case $\operatorname{grade}_{A}(\mathfrak{a}, M)=\infty$. So assume that $\mathfrak{a} M \neq M$. Then using [5. Proposition 1.2.10(a)], there is a prime ideal $\mathfrak{p}$ containing $\mathfrak{a}$ such that $\operatorname{grade}_{A}(\mathfrak{a}, M)=\operatorname{depth} M_{\mathfrak{p}}$. Hence by assumption one has $\operatorname{grade}_{A}(\mathfrak{a}, M)=\operatorname{depth} M_{\mathfrak{p}}=\operatorname{dim} R_{\mathfrak{p}}=\mathrm{ht} \mathfrak{p} \geq$ ht $\mathfrak{a}$. To prove the converse assume that $\mathfrak{p} \in \operatorname{Supp}_{A}(M)$. Then again in view of [5, Proposition 1.2.10(a)], one has $\operatorname{dim} R_{\mathfrak{p}}=\mathrm{htp} \leq \operatorname{grade}_{A}(\mathfrak{p}, M) \leq \operatorname{depth} M_{\mathfrak{p}}$. Thus $M_{\mathfrak{p}}$ is maximal Cohen-Macaulay.

Corollary 4.9. Assume that $A$ is Noetherian, and that $J \subseteq \mathrm{Nil} B$ is finitely generated as an $A$ module. Then $A \bowtie^{f} J$ is Cohen-Macaulay if and only if $A$ is Cohen-Macaulay and $J_{\mathfrak{p}}$ is maximal Cohen-Macaulay for every prime ideal $\mathfrak{p} \in \operatorname{Supp}_{A}(J)$.

The next proposition provides other sufficient and necessary condition for $A \bowtie^{f} J$ to be CohenMacaulay in the sense of ideals.

Proposition 4.10. With the notation and hypotheses of the beginning of Section 3, one has

(i) Let $\mathscr{A}$ be a non-empty class of ideals of A. Assume that $\mathrm{ht} f^{-1}(\mathfrak{q}) \leq \mathrm{ht} \mathfrak{q}$ for every $\mathfrak{q} \in$ $\operatorname{Spec}(B) \backslash V(J)$. If $A \bowtie^{f} J$ is Cohen-Macaulay (ring) in the sense of $\mathscr{A}^{e}:=\left\{\mathfrak{a}^{e} \mid \mathfrak{a} \in \mathscr{A}\right\}$, then $A$ is Cohen-Macaulay in the sense of $\mathscr{A}$ and $\mathrm{K}$. grade $\operatorname{gra}_{A}(\mathfrak{a}, J) \geq$ ht $\mathfrak{a}$ for every $\mathfrak{a} \in \mathscr{A}$.

(ii) Assume that ht $\mathscr{P} \leq$ ht $\mathscr{P}^{c}$ for every $\mathscr{P} \in \operatorname{Spec}\left(A \bowtie^{f} J\right)$, where the contraction $\mathscr{P}^{c}$ is given with respect to $\imath_{A}$. If $A$ is Cohen-Macaulay in the sense of ideals and $\mathrm{K}_{\text {. }} \operatorname{grade}_{A}(\mathfrak{a}, J) \geq$ ht $\mathfrak{a}$ for every ideal $\mathfrak{a}$ of $A$, then $A \bowtie^{f} J$ is Cohen-Macaulay (ring) in the sense of ideals.

Proof. (1) Assume that $A \bowtie^{f} J$ is Cohen-Macaulay ring in the sense of $\mathscr{A}^{e}$. In order to prove the assertion, by Proposition 4.5, it is enough for us to show that ht $\mathfrak{a}^{e} \geq$ ht $\mathfrak{a}$ for each ideal $\mathfrak{a} \in \mathscr{A}$. To 
this end, assume that $\mathfrak{a} \in \mathscr{A}$ and that $\mathscr{P}$ is a prime ideal of $A \bowtie^{f} J$ containing $\mathfrak{a}^{e}$. In view of [9, Corollaries 2.5 and 2.7], one has the following three cases to consider.

Case 1. If $\mathscr{P}=\mathfrak{p}^{\prime} f$ for some prime ideal $\mathfrak{p}$ of $A$ such that $f^{-1}(J) \nsubseteq \mathfrak{p}$, then

$$
\text { ht } \mathscr{P}=\text { ht } \mathfrak{p}^{\prime}=\operatorname{dim}\left(A \bowtie^{f} J\right)_{\mathfrak{p}^{\prime} f}=\operatorname{dim} A_{\mathfrak{p}}=\text { ht } \mathfrak{p} \geq \text { ht } \mathfrak{a},
$$

by [9. Proposition 2.9] and Lemma4.3. (1).

Case 2. If $\mathscr{P}=\mathfrak{p}^{\prime} f$ for some prime ideal $\mathfrak{p}$ of $A$ such that $f^{-1}(J) \subseteq \mathfrak{p}$, then

$$
\begin{aligned}
\text { ht } \mathscr{P} & =\mathrm{ht} \mathfrak{p}^{\prime} f \\
& =\operatorname{dim}\left(A \bowtie^{f} J\right)_{\mathfrak{p}^{\prime} f} \\
& =\operatorname{dim}\left(A_{\mathfrak{p}} \bowtie^{f_{\mathfrak{p}}} J_{S_{\mathfrak{p}}}\right) \\
& =\max \left\{\operatorname{dim} A_{\mathfrak{p}}, \operatorname{dim}\left(f_{\mathfrak{p}}\left(A_{\mathfrak{p}}\right)+J_{S_{\mathfrak{p}}}\right)\right\} \\
& \geq \operatorname{dim} A_{\mathfrak{p}} \\
& =\mathrm{ht} \mathfrak{p} \\
& \geq \mathrm{ht} \mathfrak{a},
\end{aligned}
$$

by [9. Proposition 2.9], [8, Proposition 4.1] and Lemma 4.3\} 1$)$, where $S_{\mathfrak{p}}:=f(A \backslash \mathfrak{p})+J$.

Case 3. If $\mathscr{P}=\overline{\mathfrak{q}}^{f}$ for some prime ideal $\mathfrak{q}$ of $B$, then

$$
\text { ht } \begin{aligned}
\mathscr{P} & =\text { ht } \overline{\mathfrak{q}}^{f} \\
& =\operatorname{dim}\left(A \bowtie^{f} J\right)_{\overline{\mathfrak{q}}^{f}} \\
& =\operatorname{dim} B_{\mathfrak{q}} \\
& =\text { ht } \mathfrak{q} \\
& \geq \text { ht } f^{-1}(\mathfrak{q}) \\
& \geq \text { ht } \mathfrak{a} .
\end{aligned}
$$

The third equality follows by [9. Proposition 2.9], the first inequality holds by assumption, and the second one follows by Lemma 4.3. This completes the proof of the first assertion.

(2) Assume that $A$ is Cohen-Macaulay in the sense of ideals and that $\mathrm{K}$. $\operatorname{grade}_{A}(\mathfrak{a}, J) \geq$ ht $\mathfrak{a}$ for every ideal $\mathfrak{a}$ of $A$. As indicated by [2, Theorem 3.3], it is enough to show that

$$
\text { K. } \operatorname{grade}_{A \bowtie f}\left(\mathscr{P}, A \bowtie^{f} J\right)=\text { ht } \mathscr{P}
$$

for every prime ideal $\mathscr{P}$ of $A \bowtie^{f} J$. Let $\mathscr{P}$ be a prime ideal of $A \bowtie^{f} J$. Then

$$
\text { ht } \begin{aligned}
\mathscr{P} & \leq \mathrm{ht} \mathscr{P}^{c} \\
& =\mathrm{K} \cdot \operatorname{grade}_{A}\left(\mathscr{P}^{c}, A\right) \\
& =\mathrm{K} \cdot \operatorname{grade}_{A \bowtie^{f} J}\left(\mathscr{P}^{c e}, A \bowtie^{f} J\right) \\
& \leq \mathrm{K} \cdot \operatorname{grade}_{A \bowtie^{f} J}\left(\mathscr{P}, A \bowtie^{f} J\right) \\
& \leq \mathrm{ht} \mathscr{P} .
\end{aligned}
$$

The first inequality holds by assumption, the second inequality is by [5, Proposition 9.1.2(f)], and the last one is by Lemma 3.3 (2), and the second equality follows from Lemma 3.2

We are now in a position to present our third main result.

Theorem 4.11. With the notation and hypotheses of the beginning of Section 3, the following statements hold: 
(i) Let $\mathscr{A}$ be a non-empty class of ideals of $A$. Assume that the homomorphism $f: A \rightarrow B$ satisfies the going-down property. If $A \bowtie^{f} J$ is Cohen-Macaulay (ring) in the sense of $\mathscr{A}^{e}:=\left\{\mathfrak{a}^{e} \mid \mathfrak{a} \in \mathscr{A}\right\}$, then $A$ is Cohen-Macaulay in the sense of $\mathscr{A}$ and $\mathrm{K} \cdot \operatorname{grade}_{A}(\mathfrak{a}, J) \geq$ ht $\mathfrak{a}$ for every $\mathfrak{a} \in \mathscr{A}$.

(ii) Assume that $\imath_{A}: A \rightarrow A \bowtie^{f} J$ is an integral ring extension. If $A$ is Cohen-Macaulay in the sense of ideals and $\mathrm{K}$. grade ${ }_{A}(\mathfrak{a}, J) \geq \mathrm{ht} \mathfrak{a}$ for every ideal $\mathfrak{a}$ of $A$, then $A \bowtie^{f} J$ is CohenMacaulay (ring) in the sense of ideals.

Proof. It is well-known that ht $f^{-1}(\mathfrak{q}) \leq$ ht $\mathfrak{q}$ for every $\mathfrak{q} \in \operatorname{Spec}(B)$ if the homomorphism $f: A \rightarrow B$ satisfies the going-down property by [18, Exercise 9.9]. In the light of Proposition 4.10, this proves (1). To prove (2), keeping in mind Proposition 4.10, notice that, for every $\mathscr{P} \in \operatorname{Spec}\left(A \bowtie^{f} J\right)$, the inequality ht $\mathscr{P} \leq \mathrm{ht} \mathscr{P}^{c}$ holds since $\imath_{A}: A \rightarrow A \bowtie^{f} J$ is an integral ring extension [18, Exercise 9.8], where the contraction $\mathscr{P}^{c}$ is given with respect to $\imath_{A}$.

Note that Example 4.7 also shows that we can not neglect the integral assumption in part two of the above theorem.

Example 4.12. (i) Assume that $A$ is an integral domain with $\operatorname{dim} A \leq 1$ and that $B$ is an integral domain containing $A$. Assume that $J$ is an ideal of $B$ which is finitely generated $A$-module. Hence, as in Example 4.7, one has $\mathrm{K} \cdot \operatorname{grade}_{A}(\mathfrak{a}, J)=\mathrm{ht} \mathfrak{a}$ for every proper ideal $\mathfrak{a}$ of $A$. Notice that $A$ is Cohen-Macaulay in the sense of ideals by [2, Page 2305]. Therefore one obtains that $A \bowtie^{f} J$ is Cohen-Macaulay in the sense of ideals by Theorem 4.11

(ii) To construct a concrete example for (1), set $A:=\mathbb{Q}+X \mathbb{R}[X]$, where $\mathbb{Q}$ is the field of rational numbers, $\mathbb{R}$ is the field of real numbers and $X$ is an indeterminate over $\mathbb{R}$. It is easy to see that $A$ is a one dimensional non integrally closed domain. Put $B:=A[\sqrt{2}]$, which is finitely generated as an $A$-module. Let $J$ be a finitely generated ideal of $B$. Consequently, by (1), $A \bowtie^{f} J$ is Cohen-Macaulay in the sense of ideals.

(iii) Assume that $A$ is a valuation domain, $B$ an arbitrary integral domain containing $A$ and that $J$ is an ideal of $B$. Then by [11, Corollary 4] and [12, Theorem 1], the inclusion homomorphism $f: A \hookrightarrow B$ satisfies the going-down property. Also notice, by [2, Proposition 3.12], that $A$ is Cohen-Macaulay in the sense of ideals if and only if $\operatorname{dim} A \leq 1$. Further, assume that $\operatorname{dim} A>1$. Then $A \bowtie^{f} J$ can never be Cohen-Macaulay in the sense of ideals by Theorem 4.11. In particular, the composite ring extensions $A+X B[X]$ and $A+X B[[X]]$ can never be Cohen-Macaulay in the sense of ideals.

Note that if $J$ is finitely generated as an $A$-module, then $\imath_{A}: A \rightarrow A \bowtie^{f} J$ is an integral ring extension, and that, in this case, $\mathrm{K}$. $\operatorname{grade}_{A}(\mathfrak{a}, J) \leq \mathrm{ht} \mathfrak{a}$ for every ideal $\mathfrak{a}$ of $A$ by Lemma 3.3. Hence we can make the following corollaries right away.

Corollary 4.13. Assume that the homomorphism $f: A \rightarrow B$ satisfies the going-down property and that $J$ is finitely generated as an A-module. Then $A \bowtie^{f} J$ is Cohen-Macaulay (ring) in the sense of ideals if and only if $A$ is Cohen-Macaulay in the sense of ideals and $\mathrm{K} \cdot \operatorname{grade}_{A}(\mathfrak{a}, J)=\mathrm{ht} \mathfrak{a}$ for every ideal $\mathfrak{a}$ of $A$.

Corollary 4.14. Assume that $f: A \rightarrow B$ is a monomorphism of integral domains, and $A$ is integrally closed and that $B$ is integral over $A$. Then $A \bowtie^{f} J$ is Cohen-Macaulay (ring) in the sense of ideals if and only if $A$ is Cohen-Macaulay in the sense of ideals and $\mathrm{K} \cdot \operatorname{grade}_{A}(\mathfrak{a}, J) \geq \mathrm{ht} \mathfrak{a}$ for every ideal $\mathfrak{a}$ of $A$.

Proof. By [18, Theorem 9.4], $f: A \rightarrow B$ satisfies the going-down property. Also, $l_{A}: A \rightarrow A \bowtie^{f} J$ is an integral ring extension by assumption and [8, Lemma 3.6]. 
Corollary 4.15. Assume that $f: A \rightarrow B$ is a flat and integral homomorphism. Then $A \bowtie^{f} J$ is Cohen-Macaulay (ring) in the sense of ideals if and only if $A$ is Cohen-Macaulay in the sense of ideals and $\mathrm{K} \cdot \operatorname{grade}_{A}(\mathfrak{a}, J) \geq \mathrm{ht} \mathfrak{a}$ for every ideal $\mathfrak{a}$ of $A$.

Proof. By [18, Theorem 9.5], $f: A \rightarrow B$ satisfies the going-down property. Also, $\imath_{A}: A \rightarrow A \bowtie^{f} J$ is an integral ring extension by assumption and [8, Lemma 3.6].

In concluding, we apply Corollary 4.15 on amalgamated duplication. Recall that if $f:=i d_{A}$ is the identity homomorphism on $A$, and $J$ is an ideal of $A$, then $A \bowtie J:=A \bowtie^{i d_{A}} J$ is called the amalgamated duplication of $A$ along $J$. Assume that $(A, \mathfrak{m})$ is Noetherian local. In [6, Discussion 10], assuming that $A$ is Cohen-Macaulay, D'Anna showed that $A \bowtie J$ is Cohen-Macaulay if and only if $J$ is maximal Cohen-Macaulay. Next in [21, Corollary 2.7], the authors improved D' Anna's result as $A \bowtie J$ is Cohen-Macaulay if and only if $A$ is Cohen-Macaulay and $J$ is maximal CohenMacaulay. Our final corollary generalizes these results.

Corollary 4.16. Let $J$ be an ideal of $A$. Then $A \bowtie J$ is Cohen-Macaulay (ring) in the sense of ideals if and only if $A$ is Cohen-Macaulay in the sense of ideals and $\mathrm{K} \cdot \operatorname{grade}_{A}(\mathfrak{a}, J) \geq \mathrm{ht} \mathfrak{a}$ for every ideal $\mathfrak{a}$ of $A$.

Proof. This immediately follows from Corollary 4.15, since $f=i d_{A}: A \rightarrow A$ is flat and integral.

Acknowledgements. The authors is deeply grateful to the referee for a very careful reading of the manuscript and many valuable suggestions.

\section{REFERENCES}

[1] D. D. Anderson, M. Winders, Idealization of a module, J. Commut. Algebra, 1, (2009), 3-56.

[2] M. Asgharzadeh and M. Tousi, On the notion of Cohen-Macaulayness for non-Noetherian rings, J. Algebra, 322 , (2009), 2297-2320.

[3] A. Bagheri, M. Salimi, E. Tavasoli and S. Yassemi, A construction of quasi-Gorenstein rings, J. Algebra Appl. 11, No. 1, (2012), 1250013, (9 pages).

[4] M. P. Brodmann and R. Y. Sharp, Local Cohomology: An Algebraic Introduction with Geometric Applications, Cambridge Studies in Advanced Mathematics, 136, Cambridge University Press, Cambridge, 2013.

[5] W. Bruns and J. Herzog, Cohen-Macaulay rings. Rev. ed. Cambridge Studies in Advanced Mathematics 39, Cambridge, Cambridge University Press 1998.

[6] M. D'Anna, A construction of Gorenstein rings, J. Algebra, 306, (2006), 507-519.

[7] M. D'Anna, C. A. Finocchiaro, and M. Fontana, Amalgamated algebras along an ideal, in: Commutative Algebra and Applications, Proceedings of the Fifth International Fez Conference on Commutative Algebra and Applications, Fez, Morocco, 2008, W. de Gruyter Publisher, Berlin, 2009, pp. 155-172.

[8] M. D'Anna, C. A. Finocchiaro, and M. Fontana, Properties of chains of prime ideals in an amalgamated algebra along an ideal, J. Pure Appl. Algebra, 214, (2010), 1633-1641.

[9] M. D'Anna, C. A. Finocchiaro, and M. Fontana, New algebraic properties of an amalgamated algebra along an ideal, Commun. Alg. 44, (2016), 1836-1851.

[10] M. D'Anna and M. Fontana, An amalgamated duplication of a ring along an ideal: the basic properties, J. Algebra Appl. 6, No.3, (2007), 443-459.

[11] D. E. Dobbs, On going-down for simple overrings, Proc. Amer. Math. Soc. 39, (1973), 515-519.

[12] D. E. Dobbs and I. J. Papick, On going-down for simple overrings III, Proc. Amer. Math. Soc. 54, (1976), 35-38.

[13] S. Glaz, Coherence regularity and homological dimensions of commutative fixed rings, in: Ngô Viêt Trung, Aron Simis, Guiseppe Valla (Eds.), Commutative Algebra, World Scientific, Singapore, 1992, pp. 89-106.

[14] S. Glaz, Homological dimensions of localizations of polynomial rings, in: Zero-Dimensional Commutative Rings, Knoxville, TN, 1994, in: Lect. Notes Pure Appl. Math., vol. 171, Marcel Dekker, New York, 1995, pp. 209-222.

[15] T. D. Hamilton, Weak Bourbaki unmixed rings: A step towards non-Noetherian Cohen-Macaulayness, Rocky Mountain J. Math. 34, (2004), 963-977.

[16] T. D. Hamilton and T. Marley, Non-Noetherian Cohen-Macaulay rings, J. Algebra, 307, (2007), 343-360.

[17] J. Huckaba, Commutative Rings with Zero Divisors, M. Dekker, New York, 1988. 
[18] H. Matsumura, Commutative Ring Theory, Cambridge Stud. Adv. Math., vol. 8, Cambridge University Press, 1986.

[19] M. Nagata, Local Rings, Interscience, New York, 1962.

[20] D. G. Northcott, Finite Free Resolutions, Cambridge Tracts in Math., vol. 71, 1976.

[21] P. Sahandi and N. Shirmohammadi, Notes on amalgamated duplication of a ring along an ideal, Bull. Iranian Math. Soc. 41, (2015), 749-757.

[22] P. Sahandi, N. Shirmohammadi and S. Sohrabi, Cohen-Macaulay and Gorenstein properties under the amalgamated construction, Commun. Alg. 44, (2016), 1096-1109.

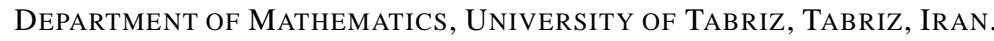

E-mail address: u.azimi@tabrizu.ac.ir

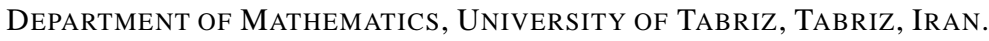

E-mail address: sahandi@ipm.ir

Department of Mathematics, University of TABriz, TABriz, Iran.

E-mail address: nshirmohammadi@yahoo.com, shirmohammadi@tabrizu.ac.ir 